\title{
La enseñanza de biomateriales en el Grado de Ingeniería de los materiales
}

\author{
Víctor manuel Pérez Puyana \\ Universidad de Sevilla. \\ Departamento de Ingeniería Química \\ vperez11@us.es \\ D.O.I.: http://dx.doi.org/10.12795/JDU.2018.i01.31 \\ Pp.: 549-565
}

\section{Resumen}

Se realizó un ciclo de mejora en la asignatura obligatoria Biomateriales que se imparte en los siguientes grados: Doble Grado en Química e Ingeniería de Materiales, Doble Grado en Física e Ingeniería de Materiales y Grado en Ingeniería de Materiales. La metodología llevada a cabo consiste en el análisis de un problema que se utiliza como punto de partida para el desarrollo de las distintas actividades. Los resultados reflejan un desarrollo en el aprendizaje de los alumnos, así como una valoración bastante positiva de ellos sobre esta metodología innovadora que se ha llevado a cabo, con una valoración media de 4.6 (sobre 5).

Palabras clave: Biomateriales, Grado en Ingeniería de Materiales, Docencia Universitaria, Experimentación Docente Universitaria, Aprendizaje basado en problemas. 


\section{Breve descripción del contexto de la intervención}

El ciclo de mejora se aplica en la asignatura de Biomateriales, asignatura que se imparte en la Facultad de Física para los grados: Doble Grado en Química e Ingeniería de Materiales, Doble Grado en Física e Ingeniería de Materiales y Grado en Ingeniería de Materiales. Se contempla como una de las asignaturas más específicas de la rama de la Ingeniería de Materiales, de ahí su importancia.

Este ciclo de mejora se desarrolla concretamente en el tema de Ingeniería de Tejidos (tema número 14, que se imparte entre los temas de Biomateriales Biológicos y Biocompatibilidad de Biomateriales) dentro del primer bloque que trata sobre los distintos tipos de biomateriales. Las sesiones que han sido centro del ciclo de mejora fueron las horas teóricas de los lunes, martes y jueves. En total, el ciclo tiene una duración de 6 sesiones de una hora en un grupo de 40 alumnos de los tres grados expuestos.

Los grandes objetivos de la asignatura son: conocimiento de diferentes biomateriales y saber evaluar las principales propiedades que deben cumplir los biomateriales, relacionándolos con distintas aplicaciones.

\section{Diseño del Ciclo de Mejora}

\section{Mapa de contenidos}

Es importante realizar un mapa de los contenidos pues se trata de un instrumento que pone de manifiesto las conexiones que establecemos entre los conocimientos que van a ser objeto de enseñanza. Además permite al docente establecer de manera global los contenidos que se van a trabajar, de forma que se pueda organizar mejor la docencia. 


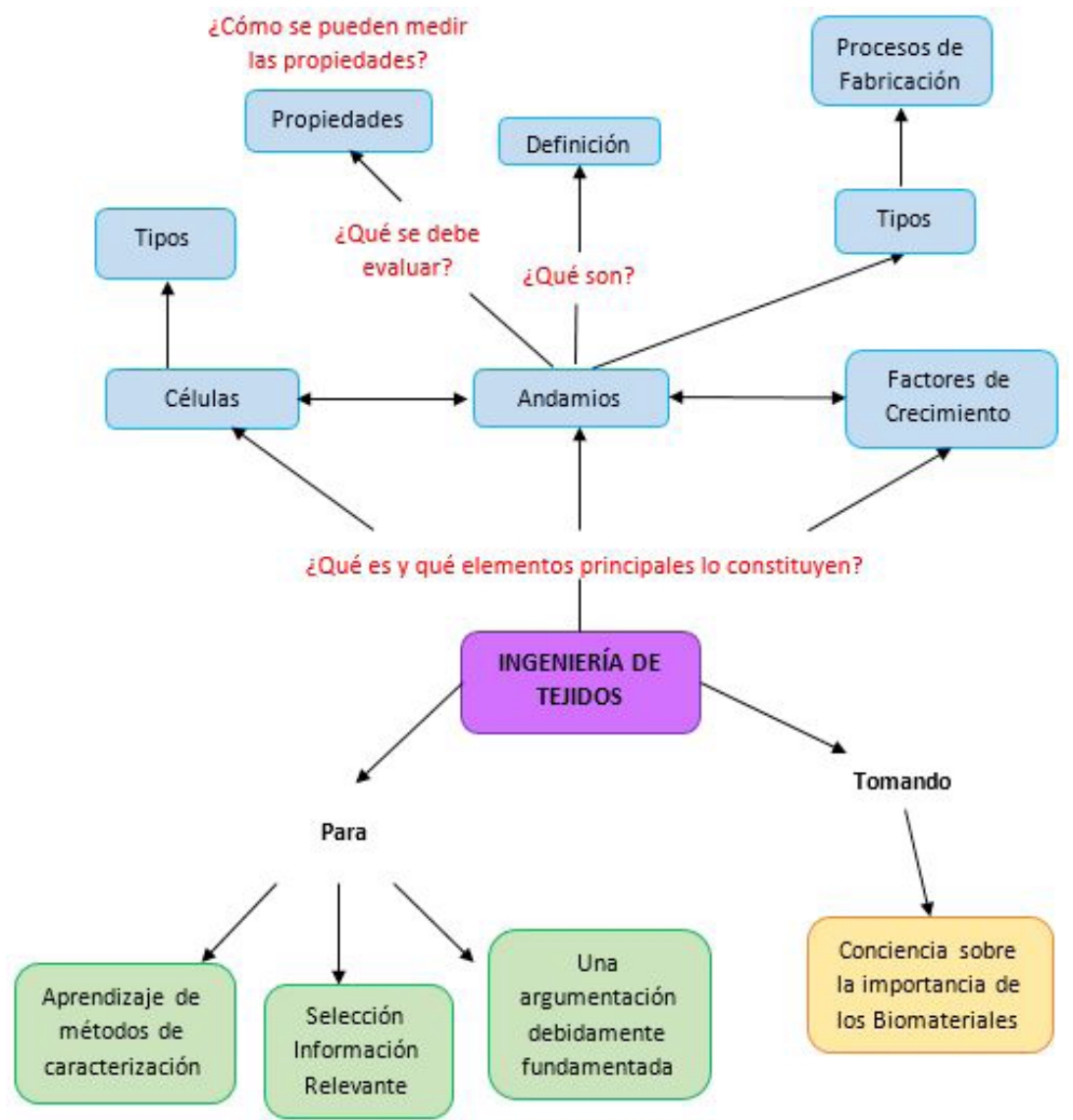

Figura 1. Mapa de contenidos del tema "Ingeniería de Tejidos".

La Figura 1 muestra el mapa de contenidos utilizado en este ciclo de mejora. Encontramos diferentes partes en dicho mapa. En el centro encontramos el concepto general sobre el que se va a trabajar y a su alrededor encontramos 3 ramas diferentes: la rama de color azul hace referencia a aquellos contenidos conceptuales que se van a trabajar, mientras que en color verde y naranja se encuentran los contenidos procedimentales y actitudinales, respectivamente, que se van a poner en juego. Además, en color rojo aparecen preguntas clave que ayudan a dirigir la docencia de aquellos contenidos de mayor relevancia en el tema. Los contenidos que se van a abordar permiten conectar

Jornadas de Formación e Innovación Docente del Profesorado I № 1 (2018)
Esta obra se distribuye con la licencia Creative Commons Reconocimiento-NoComercial-SinObraDerivada Internacional (CC BY-NC-ND 4.0.) 
esta segunda parte de la asignatura con lo que han visto inicialmente, además de lo estudiado en otras asignaturas de cursos anteriores, por lo que supone un punto de inflexión acerca de los conocimientos que han ido adquiriendo durante sus estudios.

\section{Modelo metodológico y secuencia de actividades}

El ciclo de mejora se inspira en el modelo metodológico expuesto en el Capítulo 2 del libro Enseñanza Universitaria: Cómo mejorarla (Porlán, 2017). Las etapas en las que se concreta pueden verse en la Figura 2.

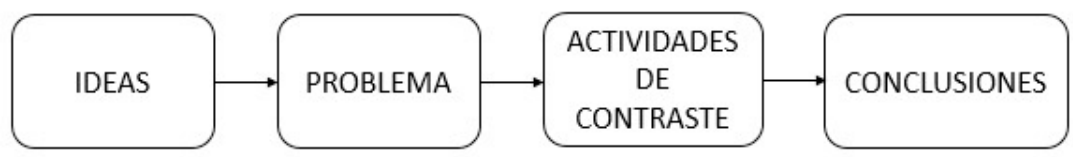

Figura 2. Etapas del modelo metodológico empleado en el Ciclo de Mejora.

La primera fase va encaminada a la exploración de las ideas iniciales de los alumnos para ver el punto de partida de la clase y así poder reorientar la docencia en función de los resultados obtenidos. A continuación se procede al planteamiento del problema, buscando la curiosidad en los alumnos, de forma que les motive para conseguir una adecuada actitud de cara al aprendizaje. Tras esto, se procede a la realización de actividades de contraste en las que los protagonistas son los propios alumnos. Finalmente, se procede a la elaboración de conclusiones por parte de los alumnos sobre lo que han trabajado. La secuencia de actividades es la que se expone a continuación.

Realización del cuestionario inicial de ideas iniciales (20 min.)

En esta primera actividad los estudiantes realizan el cuestionario de ideas iniciales (Tabla 1) que va a servir al docente para ver el punto de partida de sus alumnos.

Como recurso se necesitan copias del cuestionario.

Jornadas de Formación e Innovación Docente del Profesorado | № 1 (2018) Esta obra se distribuye con la licencia Creative Commons 
Tabla 1. Preguntas que conforman el cuestionario de ideas iniciales.

\begin{tabular}{|l|l|}
\hline \multicolumn{2}{|c|}{ Cuestionario de ideas iniciales } \\
\hline 1a Pregunta & $\begin{array}{l}\text { El concepto de Ingeniería de Tejidos ha tenido numerosas } \\
\text { definiciones en su corto tiempo de estudio, ¿por qué crees } \\
\text { que es así?, ¿cómo lo definirías tú? }\end{array}$ \\
\hline 2a- Pregunta & $\begin{array}{l}\text { Si tuvieras que llevar a cabo una investigación en dicho } \\
\text { campo de trabajo, ¿sobre qué lo harías y qué aspectos de } \\
\text { interés crees que serían los objetivos clave a cumplir? }\end{array}$ \\
\hline 3a Pregunta & $\begin{array}{l}\text { Si tuvieras que seleccionar un andamio para su aplicación } \\
\text { en Ingeniería de Tejidos, ¿en qué te fijarías o qué conside- } \\
\text { rarías como importante? }\end{array}$ \\
\hline 4a Pregunta & $\begin{array}{l}\text { Señala la/s posible/s respuesta/s verdadera/s. Justifícalo } \\
\text { brevemente: } \\
\text { a. Todos los andamios son biomateriales útiles para Inge- } \\
\text { niería de Tejidos } \\
\text { b. Todos los biomateriales son andamios } \\
\text { c. El colágeno es un polímero sintético }\end{array}$ \\
\hline
\end{tabular}
$\min$.)

Ejemplo real relacionado con el tema a impartir (40

El peso de esta actividad recae sobre todo en el profesor. Esta primera actividad se va a realizar durante la primera sesión y va encaminada al planteamiento del problema/idea central, de forma que se contextualiza el tema y se intenta promover la curiosidad de los alumnos. La actividad consiste en la descripción de un caso real que implique la aplicación de un biomaterial para tratar el problema.

Como recursos se necesitan copias de nota de prensa y proyector.

Los contenidos que se van a tratar son de carácter procedimental y actitudinal. Los contenidos actitudinales son la adaptación a nuevas situaciones y la actitud crítica.

Charla de una experta (1 h.)

Esta actividad es una continuación de la actividad anterior y de nuevo conlleva una participación activa de los alumnos. Dicha actividad va encaminada a la toma de 
conciencia de los alumnos mediante una charla impartida por una experta (investigadora del área de Ingeniería de Tejidos), mediante la descripción de aplicaciones y ejemplos reales que se están desarrollando en el laboratorio.

La actividad consiste en la exposición de un experto/ investigador del campo de la Ingeniería de Tejidos, describiendo las propiedades y aplicaciones reales de los biomateriales en esa área. Conforme se va avanzando en la charla, el experto lanzará preguntas a los alumnos de manera que haya una cooperación/interacción entre ellos, es decir, esta actividad va a desembocar en una actividad grupal en la que colaboren y tengan protagonismo los propios alumnos. Para ello, se estimulará a los alumnos a exponer todas las dudas o sugerencias que les hayan podido surgir sobre los distintos contenidos.

Como recursos se necesita pizarra y proyector.

Se trata de una actividad muy completa ya que los contenidos que se tratan son de carácter conceptual (contenidos teóricos sobre el tema), procedimental (enfocado en cómo pueden usarse esos conocimientos para tratar las aplicaciones comentadas, formas de razonar sobre el tema) y actitudinal (punto de vista y sensibilidad hacia el tema). Así:

- Contenido conceptual: Conocimiento y comprensión de la tecnología y aplicaciones de los materiales.

- Contenido procedimental: Capacidad de diseño y selección de materiales para aplicaciones específicas.

- Contenido actitudinal: Conciencia sobre la importancia de los biomateriales.

Debate grupal sobre un caso real (45 min.)

Esta actividad es un debate grupal entre los alumnos. Es una actividad puramente de contraste, analizando los distintos puntos de vista de los alumnos, de forma que ellos planteen su punto de vista respecto a las opiniones de sus compañeros. El profesor actuará como un mero moderador controlando el debate, esto es, dando paso a cada 
uno de los estudiantes que desee intervenir y fomentando la participación de aquellos que no tengan tanta iniciativa.

La actividad consiste en un debate grupal sobre dos notas de prensa que han aparecido en periódicos de tirada nacional como El País y El Mundo en el año 2000 y 2015, respectivamente. En ellos se lleva a cabo una descripción del campo de estudio que estamos desarrollando en las sesiones (Ingeniería de Tejidos) detallando un caso de éxito que ha tenido lugar en EEUU. Por tanto, la actividad se plantea desde una perspectiva divulgativa, es decir, tras la lectura de ambas noticias, los propios alumnos son los que van a describir y analizar los contenidos que aparecen en los artículos, dando una opinión personal final sobre qué les ha parecido.

Como recursos, las copias de las notas de prensa.

Los contenidos que se tratan son de carácter procedimental y actitudinal:

- Contenido procedimental: Toma de decisiones, capacidad de trabajo interdisciplinar.

- Contenido actitudinal: Actitud crítica y saber escuchar otras opiniones.

Exposición teórica (planteamiento de dudas y preguntas de los alumnos) (2 h.)

Esta actividad conlleva una interacción activa entre el docente y los alumnos. Va encaminada a la descripción de conceptos teóricos que se van a poner en juego durante el transcurso del tema. La actividad consiste en la exposición teórica del tema "Ingeniería de Tejidos", describiendo los tipos, propiedades y formas de procesado de este tipo de materiales. Conforme se va avanzando en los contenidos teóricos, el profesor lanzará preguntas a los alumnos de manera que haya una cooperación/interacción entre ellos y con el propio profesor; es decir, esta actividad va a comenzar de manera individual por parte del profesor y va a desembocar en una actividad grupal en la que colaboren y tengan bastante protagonismo los propios alumnos. 
Para ello, se fomentará (con preguntas del profesor) que los alumnos expongan todas las dudas que puedan surgir sobre los distintos contenidos. Asimismo, se harán continuas referencias/menciones al artículo de prensa donde aparece tanto la idea central sobre la que se centran todos los contenidos del tema como los elementos que desembocan de él. De esta forma, se consigue fomentar un clima distendido para la adquisición de conocimiento mediante la exposición del tema.

Como recursos se necesita proyector y presentación en PowerPoint de apoyo.

Los contenidos que se van a tratar son de carácter conceptual (contenidos teóricos sobre el tema) y procedimental (enfocado en cómo pueden usarse esos conocimientos para tratar una aplicación específica):

- Contenido conceptual: Conocimiento y comprensión del comportamiento químico y biológico de los materiales.

- Contenido procedimental: Capacidad de diseño y selección de materiales para aplicaciones específicas.

Debate final sobre un caso hipotético y Elaboración de conclusiones (40 min.)

La actividad consiste un debate grupal en el que se trata de volver y enlazar con la idea central/principal que se expuso al comienzo del ciclo de mejora. Los alumnos debaten destacando sus soluciones propuestas (señalando los aspectos positivos). Por tanto, la actividad se plantea desde la perspectiva profesional (en la pizarra se colocan 4 empresas con 4 biomateriales con características diferentes, con una tabla, y los alumnos deberán decantarse por una opción u otra y justificar su decisión apoyándose en los conocimientos adquiridos durante las sesiones). En definitiva, los propios alumnos son los que van a seleccionar una opción posible consensuando con el resto de sus compañeros su opinión. Tras eso, se dejará tiempo a los alumnos para que reflexionen (e incluso que discutan 
en pequeños grupos posibles soluciones). Para finalizar esta actividad, se busca la elaboración de conclusiones por parte de los alumnos sobre las soluciones planteadas, destacando la que ellos consideran más adecuada. Así, se cierra el ciclo volviendo al planteamiento de partida y relacionándolo con los conocimientos que han ido adquiriendo durante la secuencia de las distintas actividades.

Como recurso simplemente se utiliza la pizarra.

En esta actividad se tratan los 3 tipos de contenidos: conceptuales (recordando los conceptos teóricos de las actividades anteriores), procedimentales (destacando la forma de pensar y justificar que su opinión era la más adecuada) y actitudinales (enfatizando la forma en la que se ha buscado y argumentado críticamente dicha solución):

- Contenido conceptual: Conocimiento y comprensión de los biomateriales.

- Contenido procedimental: Capacidad de análisis de distintos materiales según sus aplicaciones.

- Contenido actitudinal: Actitud de trabajo en grupo y actitud crítica ante comentarios de otros compañeros.

Realización del cuestionario final de ideas (20 min.)

Finalmente, los estudiantes vuelven a realizar el mismo cuestionario que realizaron inicialmente, de forma que el docente puede evaluar la evolución de sus alumnos tras el desarrollo de las sesiones planificadas.

Como recurso se necesitan copias del cuestionario.

\section{Cuestionario inicial-final}

El instrumento para analizar los conocimientos iniciales de los estudiantes en el tema sobre el que se va a aplicar el ciclo de mejora -y posteriormente como cuestionario final- consiste en un cuestionario de 4 preguntas que van desde aspectos más generales hasta otros más específicos que se pretende trabajar (Tabla 1). 
Dicho cuestionario presenta -como se vio-cuatro preguntas. Tres de ellas son preguntas cuya respuesta es abierta para dejar libertad a los alumnos para expresarse libremente (exponiendo sus ideas). La última cuestión es una pregunta de tipo Verdadero/Falso. Cierto es que en este tipo de cuestionarios hay que evitar las preguntas dicotómicas pero he considerado adecuado el utilizar una pregunta tipo test de rápida contestación mediante la cual puedo evaluar de manera más objetiva los conocimientos de los alumnos. Las dos primeras preguntas se centran en contenidos de carácter general, es decir, los principales que deben adquirir los alumnos tras llevar a cabo la secuencia de actividades prevista para el tema de "Ingeniería de Tejidos". La tercera pregunta está relacionada con conceptos más específicos que implican un mayor dominio de la materia y, por último, la cuarta pregunta (de V o F) permite al docente evaluar si los alumnos relacionan los contenidos del tema a impartir con conceptos y contenidos previamente adquiridos en temas anteriores.

\section{Aplicación del Ciclo de Mejora}

\section{Relato resumido de las sesiones}

El uso del diario del profesor ha permitido anotar todas las curiosidades y dificultades observadas durante el desarrollo del ciclo de mejora. Lo primero que destaco es el leve rechazo inicial de gran parte del alumnado al cuestionario de ideas iniciales ya que lo asemejaban a un examen que les iba a contar para su nota en la asignatura; sin embargo, una vez aclarado y dejado claro que iba a ser anónimo, lo completaron sin problema alguno. A pesar del pequeño percance inicial, la realización del cuestionario se llevó a cabo en el tiempo previsto. En cuanto a su experiencia con la investigadora experta del tema que 
se impartió, destaco las impresiones positivas de los estudiantes, quedando gratamente satisfechos con dicha actividad.

Respecto a la actividad referente a la puesta en común de los artículos de prensa de tirada nacional (El País y El Mundo), la duración de la actividad superó al tiempo preestablecido debido a la falta de iniciativa inicial de los alumnos. Una vez se ha ido desarrollando la actividad, la participación de los alumnos ha ido mejorando. Conectando con esta actividad y con los resultados del cuestionario inicial de los alumnos, decidí alargar intencionalmente la actividad de explicación teórica llevada a cabo durante la cuarta y quinta sesión ya que, tal y como comprobé en los cuestionarios iniciales, había una gran parte de la clase en el nivel inicial, esto es, que no presentaban conocimientos apenas sobre el tema (esto me permitió lanzar preguntas a los alumnos de manera que hubiera una cooperación/interacción entre ellos, haciendo numerosas alusiones a los artículos de prensa).

Finalmente, en la última sesión se procedió a la realización del cuestionario final de ideas, así como un debate acerca de un caso hipotético que engloba todos los conocimientos puestos en juego durante las sesiones anteriores. En ambos casos destaco la participación activa de la mayoría de la clase, y debido al entusiasmo mostrado por la clase decidí alargar un poco más el debate.

\section{Evaluación del aprendizaje de los estudiantes}

A partir de los resultados obtenidos en el cuestionario, en sus pases inicial y final, he podido analizar los conocimientos de los que partía la clase de forma que pude reorganizar la docencia a impartir para optimizar el tiempo lo mejor posible. Para ello elaboré una escalera de aprendizaje en la que situé a los alumnos en función de sus conocimientos. Dicha escalera, así como sus distintos escalones se han establecido de manera común para la 
valoración del conocimiento mostrado en las respuestas de cada una de las 4 preguntas que se han incluido en el cuestionario. Los distintos niveles que se establecieron son los siguientes:

- Nivel 1. No presenta conocimientos o conocimientos muy básicos sobre el tema.

- Nivel 2. Presenta ciertos conocimientos pero no sabe aplicarlos adecuadamente.

- Nivel 3. Maneja conceptos relacionados con el tema pero tiene fallos de concepto.

- Nivel 4. Domina los contenidos y relaciona los conceptos implicados con los que ya disponían con anterioridad (respuestas de mayor complejidad).

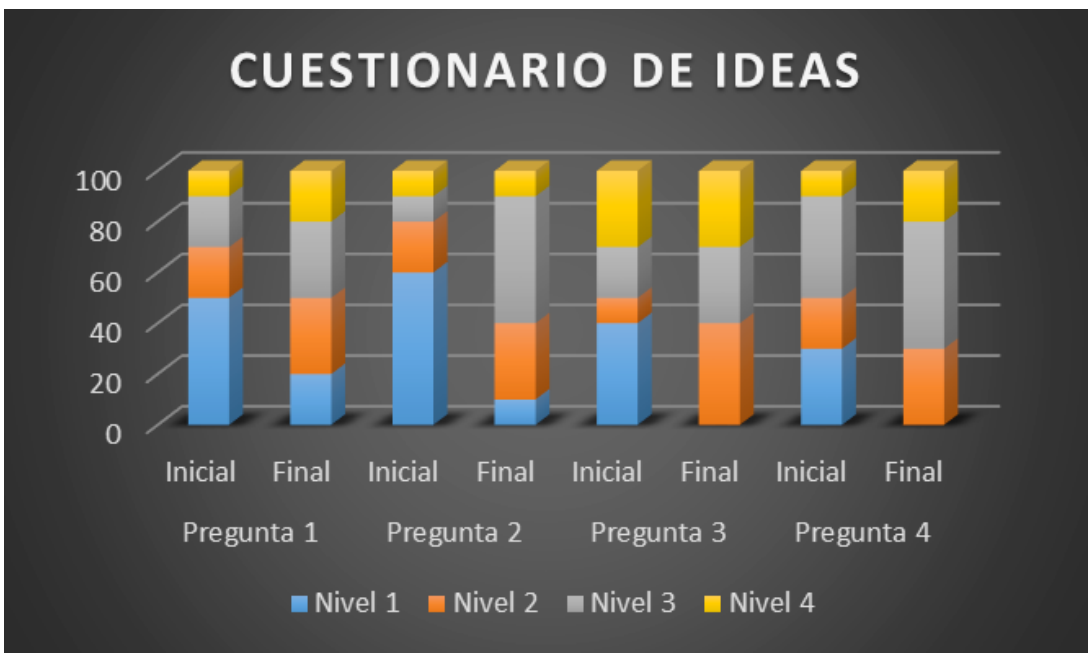

Figura 3. Niveles de conocimiento iniciales y finales del alumnado.

El análisis de los resultados puede llevarse a cabo teniendo en cuenta el nivel de la clase en general. En la Figura 3 se representan los niveles de conocimiento iniciales y finales mostrados en las respuestas del alumnado a las 4 preguntas citadas, lo cual refleja los conocimientos de que dispone la clase sobre los aspectos más relevantes del tema de estudio. En cuanto a los conocimientos iniciales podemos destacar que se trata de un grupo heterogéneo, ya que hay alumnos distribuidos en todos los niveles

Jornadas de Formación e Innovación Docente del Profesorado | № 1 (2018) Esta obra se distribuye con la licencia Creative Commons 
establecidos. En todo caso, es importante destacar que, en el cuestionario inicial, la mayor parte de la clase (más de un $70 \%$ ) se encuentra en los niveles 1 y 2 , lo cual indica que los alumnos no presentan demasiados conocimientos respecto al problema a tratar.

Tras la realización del ciclo de mejora, los alumnos volvieron a realizar el mismo cuestionario que realizaron inicialmente. Los resultados reflejan un avance global de la clase, pues la mayoría ha alcanzado los niveles superiores, reflejo de que han interiorizado los conocimientos impartidos. Es importante destacar que no todos los alumnos se encuentran en el mismo nivel, es decir, en el mismo tramo de la escalera de aprendizaje. Además, la idea de escalera lleva asociado la determinación de obstáculos, pues si no se modifica la planificación docente en función de los mayores obstáculos que presente la clase, los cuestionarios pierden toda utilidad. Así:

- El primer obstáculo está relacionado con el poco interés que pueden presentar algunos alumnos por la asignatura; para el cual hay que prestar especial atención y redirigir la docencia de forma que se consiga motivar a dichos alumnos (paso del escalón 1 al 2).

- El segundo obstáculo está relacionado con la dificultad que presentan los alumnos para aplicar los conocimientos que han aprendido (falta de aplicabilidad), lo que implica una falta de interiorización de dichos contenidos (del escalón 2 al 3).

- Por último, el tercer obstáculo está relacionado con la falta de visión global de la asignatura. Los alumnos presentan conocimientos pero no son capaces de relacionarlos entre ellos y con los que disponían anteriormente (del escalón 3 al 4). 


\section{Evaluación del Ciclo de Mejora}

\section{Valoración de la docencia}

La evaluación de la docencia puede llevarse a cabo de diferentes maneras (con diferentes instrumentos). En este caso particular, se utilizan dos instrumentos diferentes ya que, por un lado, se lleva a cabo la observación del desarrollo de cada sesión mediante las anotaciones del diario de clase, lo que permite evaluar la metodología aplicada -como se ha hecho anteriormente-, y, por otro lado, también se motivó a los alumnos a realizar una encuesta en la que valoraron de 1 a 5 (1: nada satisfecho y 5: totalmente satisfecho) la práctica docente. Los resultados obtenidos (Tabla 2) reflejan que el grado de satisfacción para el alumnado encuestado sobre el desarrollo de las sesiones del ciclo de mejora ha sido muy positivo.

Tabla 2. Encuesta y puntuación media de los estudiantes a distintos aspectos de la docencia.

\begin{tabular}{|ll|}
\hline \multicolumn{2}{|c|}{ Encuesta de valoración de la docencia } \\
\hline \multicolumn{2}{|c|}{ 1: Nada Satisfecho 5: Totalmente Satisfecho } \\
\hline La organización me ha parecido adecuada. & 4 \\
\hline El desarrollo de la docencia me ha resultado motivador & 4 \\
\hline Los conceptos puestos en juego han quedado claros & 5 \\
\hline Ha habido una buena interacción alumno-profesor & 5 \\
\hline La interacción con el resto de mis compañeros ha sido adecuada & 5 \\
\hline
\end{tabular}

\section{Cuestiones a mantener y cambios a introducir}

Valoro muy positivamente esta experiencia por los avances logrados, sobre todo, gracias a las actividades en las que ha participado la clase en conjunto, como el 
debate, pues gracias a ellas he conseguido atraer el interés de los estudiantes, consiguiendo una mayor participación e interacción durante el desarrollo de las actividades. Además, la ventaja principal de usar este tipo de metodología es que se consigue mejorar la participación de los estudiantes. Sin embargo, el inconveniente principal es el tiempo que requiere el llevar a cabo esta secuencia de actividades de la mejor manera posible.

Por tanto, opino que es necesaria una mayor optimización de los tiempos de realización de las distintas actividades propuestas, lo que permitiría poder llevar a cabo la práctica de una manera más eficiente de cara al aprendizaje de los alumnos. Por ende, propongo como propuesta de mejora el uso de aplicaciones informáticas (Socrative o Kahoot!) para llevar a cabo el proceso de conocimiento de las ideas iniciales de los alumnos, de forma que se pueda agilizar dicho proceso, a la vez que se promueve la motivación de los alumnos, pues a veces el hecho de ver/realizar un cuestionario les parece similar a un examen.

\section{Aspectos a incorporar a la práctica docente}

Los cambios que me gustaría incluir de manera habitual en mis futuras clases es la realización de debates pues creo que permite a los alumnos expresarse libremente dando su opinión (fundamentada por datos), lo cual me permitiría conocer el nivel de implicación de los estudiantes con la asignatura, así como los conocimientos que van adquiriendo. Además, me he percatado de que hay que controlar muy bien las tareas grupales, como los debates, para hacer hincapié en aquellos aspectos que se quieren destacar así como marcar las pautas que permiten un buen desarrollo de los mismos (respeto por las opiniones de otros compañeros, turnos de palabra, etc.). 


\section{Principios didácticos argumentados}

De todos aquellos principios didácticos que tuve en cuenta para la aplicación y desarrollo del ciclo de mejora, destaco 3 que considero como los pilares fundamentales, que son:

Participación activa del alumnado. Los métodos de aprendizaje que destacan son aquellos que fomentan al alumno a tener un papel activo/protagonista durante el proceso de enseñanza-aprendizaje (Fernández, 2006; Gureckis y Markant, 2012).

Aprendizaje colaborativo. Las sesiones y actividades planteadas han permitido que los estudiantes interactúen entre sí permitiendo que puedan aprender unos de otros (Mari Ytarte et al., 2016; Vallet-Bellmunt et al., 2017).

Razonamiento crítico. Se intentó indagar en la capacidad de los estudiantes para replantearse todo lo que le rodea, de forma que sean capaces de dar una opinión razonada y argumentada (López, 2011).

Motivación. Pilar fundamental sobre el cual se sustenta todo el ciclo de mejora.

A modo de conclusión, opino que toda materia es como el universo, infinito, por lo que el desarrollo de un buen modelo metodológico es esencial, ya que hay que definir qué impartir, y más importante aún, cómo impartirlo.

Jornadas de Formación e Innovación Docente del Profesorado | № 1 (2018) Esta obra se distribuye con la licencia Creative Commons 


\section{Referencias bibliográficas}

De Alba, N. y Porlán, R. (2017). La metodología de enseñanza. En R. Porlán (coord.), Enseñanza universitaria. Cómo mejorarla (pp. 37-54). Madrid: Morata.

Gureckis, T. M., Markant, D. B. (2012). Self-Directed Learning: A Cognitive and Computational Perspective. Perspectives on Psychological Science, 7(5), 464-481.

Fernández, M. R. (2006). Reseña de Metodología participativa en la Enseñanza Universitaria de Fernando López Noguero. Revista Interuniversitaria de Formación del Profesorado, 20(3), 313-316.

López, J. I. (2011). Un giro copernicano en la enseñanza universitaria: formación por competencias. Revista de Educación, 356, 279-301.

Marí Ytarte, R. M., Moreno, R., Hipólito, N. (2016). Educación y ciudadanía. Propuestas educativas desde la controversia. Foro de Educación, 14(20), 49-69.

Vallet-Bellmunt, T., Rivera-Torres, P., Vallet-Bellmunt, I. y Vallet-Belmunt, A. (2017). Aprendizaje cooperativo, aprendizaje percibido y rendimiento académico de la enseñanza de marketing. Educación XX1, 20(1), 277-297.

Jornadas de Formación e Innovación Docente del Profesorado | № 1 (2018) Esta obra se distribuye con la licencia Creative Commons 